\title{
FUNGAL DIVERSITY IN FLORAL AND HONEYDEW HONEYS
}

\author{
D. Magyar ${ }^{1}$, A. Mura-Mészáros ${ }^{2}$ and F. Grillenzoni ${ }^{3}$ \\ ${ }^{1}$ Department of Air Hygiene and Aerobiology, National Public Health Centre \\ H-1097 Budapest, Gyáli út 2-6, Hungary; E-mail: magyar.donat@gmail.com \\ ${ }^{2}$ Institute for Biology, Faculty of Veterinary Science, Szent István University \\ Budapest, Hungary \\ (Department of Cell and Molecular Biology, Leibniz Institute for Natural Product \\ Research and Infection Biology Hans Knöll Institute, Jena, Germany) \\ ${ }^{3}$ Honeybee and Silkworm Research Unit, Consiglio per la Ricerca in Agricoltura e l'analisi \\ dell'economia Agraria, Bologna, Italy
}

(Received 11 April, 2015; Accepted 15 November, 2015)

Studying fungal diversity in various environmental samples provides us with valuable knowledge about the occurrence of fungi of medical and ecological importance. Moreover, fungal composition may also characterise well the botanical and geographical source of food products, such as the origin of the spore enriched honeydew honeys. Thereby, we identified a wide spectrum of fungi found in 100 of honey samples from various geographical sources - most of them were from Italy, Greece and Hungary. Our honeydew honeys had a higher mean of the number of spore types found in them than floral honeys had. Statistically significant differences in diversity were found regarding the botanical source $\left(p=1.29 \times 10^{-9}\right)$ and the climatic classification $\left(p=2.28 \times 10^{-2}\right)$ according to KruskalWallis rank sum tests. Most frequently encountered genera included ubiquitous saprotrophic species (Alternaria, Cladosporium, Epicoccum nigrum, Stemphylium), both in floral and honeydew honeys. On the other hand, certain sooty moulds like Aureobasidium pullulans, Tripospermum and Capnobotrys were rather present in different types of honeydew honeys. Metschnikowia reukaufii, the nectar inhabiting yeast reached considerably high quantities in floral honey samples. Present findings encourage further studies on quantifying the occurrence and the indicator value of specific fungal elements in honey, concerning its origin.

Key words: botanical origin, diversity, fungi, honey, honeydew

\section{INTRODUCTION}

The diversity of fungal species is an intensively studied subject because of ecological, phytopathological and pharmaceutical importance of fungi. Morphological identification of species is often used to detect various groups of fungi, e.g. allergenic and phytopathogenic fungi in air samples, Ingoldian fungi in stream water, fossilised spores in historical or forensic samples. Hon- 
eys, especially those of honeydew origin are also rich in fungal spores (Dimou et al. 2006, Magyar et al. 2005, Pérez-Atanes et al. 2001, Seijo et al. 2011, Zander 1935). Spore content of honeys arose increasing interest, because it can help to determine their source. Knowing that fungi frequently live in a strong association with plants, different fungal species assemblages might be as typical of the honey as the spectrum of pollen grains.

Honeydew elements, namely fungal spores, hyphae fragments and algae are used as indicators of honeydew origin of honeys (Louveaux et al. 1978), due to their frequent accumulation in honeydew. Honeydew is the sugary secretion of phytophagous insects (i.e. Rinchota: Homoptera, Magyar et al. 2005). It is collected by bees mainly from late summer till September when there is a limited source of nectariferous flowers (Persano-Oddo et al. 2000). Honeydew honeys often reach a higher price than other types of honey speaking mainly of Austria, Switzerland and Germany (Bogdanov and Martin 2002). In comparison with floral honeys, honeydew honeys have a higher mineral (González-Miret et al. 2005) and oligosaccharide content (Földházi 1994). Certain countries' total honey production, for example that of Greece, comes predominantly from honeydew honey (Thrasyvoulou and Manikis 1995). Thereby, the reliable identification of such honeys is of special commercial interest.

During traditional melissopalynological analysis, honeydew elements are quantified next to pollens. A careful differentiation between the pollen types of nectariferous and those of anemophilous plant species, as well as the ability to separate underrepresented elements from overrepresented ones, are important skills for an accurate honey validation process. As microscopical elements are direct biological indicators of the honey source and their analysis has a good reproducibility (Louveaux et al. 1978), their classification is surely useful. However, identification of fungal particles accounting for the majority of counted honeydew elements, is regrettably neglected. The lack of knowledge about fungal species occurring in honey samples, represented by their spores is a significant loss of information in honey analysis.

Therefore, our aim was to characterise fungal content in a collection of honey samples from various countries and botanical sources.

\section{MATERIALS AND METHODS}

We analysed a total of 100 samples (Table 1) from three continents (Fig. 1) and 21 countries (most of them were from Italy, Greece and Hungary). Thirtyone out of them were labelled as floral honey, while 62 of them as honeydew honey. Their botanical origin was previously identified using standard melissopalynological methods and guidelines described by Louveaux et al. 
1978, Persano-Oddo and d'Albore 1989, Persano-Oddo et al. 2007, Sabatini et al. 2007 in the National Institute for Apiculture, Bologna. Seven samples were collected without pre-specified botanical origin.

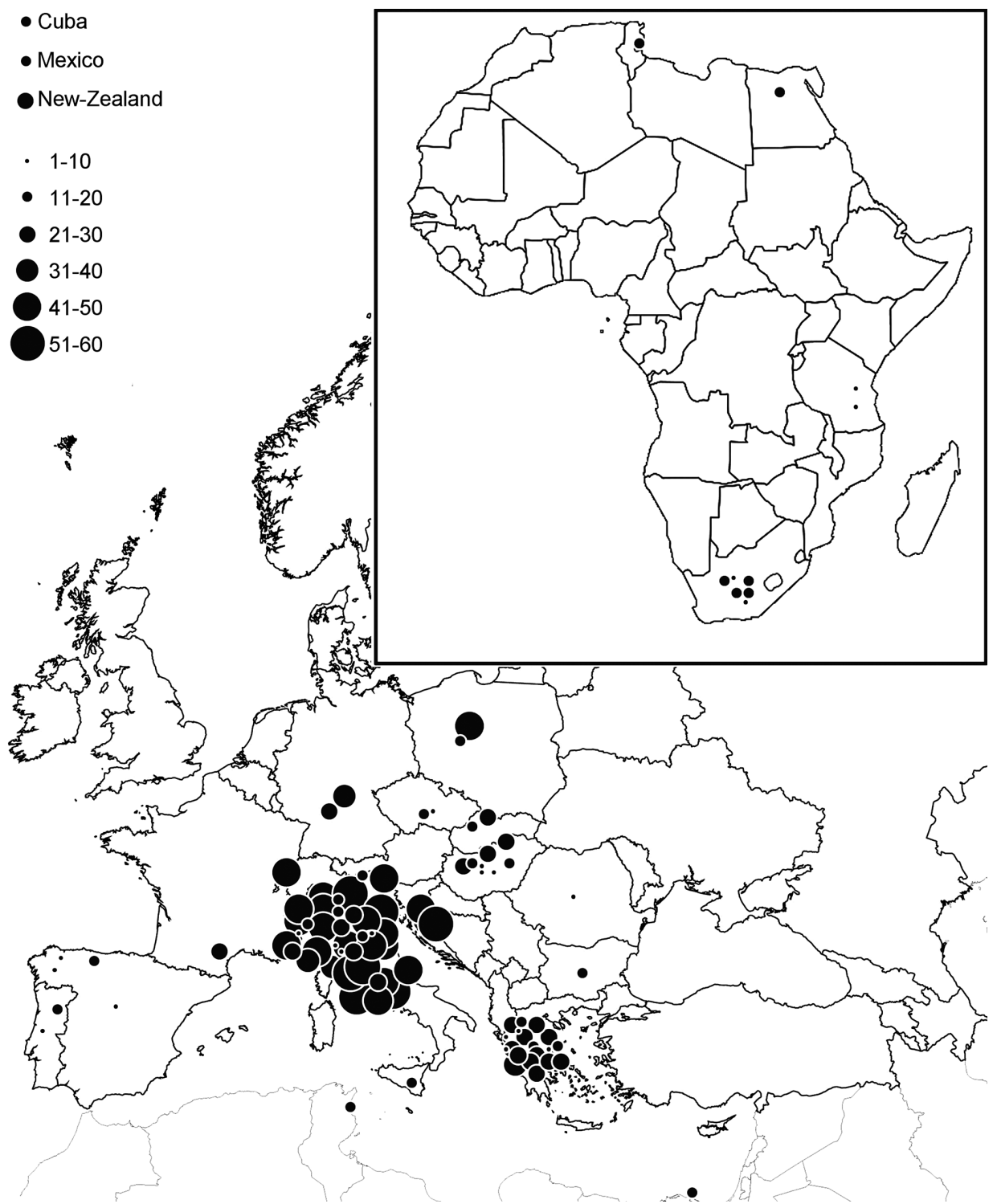

Fig. 1. Map showing all the locations, where samples were collected from. Samples are signified by spots and the diameter of each spot is proportional to the number of spore types found in the respective honey as indicated by the legend 
Table 1

List and details of examined honey samples. Abbreviations: $\mathrm{fl}=\mathrm{floral}, \mathrm{hd}=$ honeydew, unk $=$ unknown, WSC $=$ warm summer continental, $\mathrm{MT}=$ maritime temperate, $\mathrm{MTN}=$ Mediterranean, $\mathrm{WT}=$ warm temperate, $\mathrm{UD}=$ undefined

\begin{tabular}{|c|c|c|c|c|c|}
\hline $\begin{array}{l}\text { Ref. } \\
\text { no. }\end{array}$ & $\begin{array}{l}\text { Honey } \\
\text { type }\end{array}$ & Locality & $\begin{array}{l}\text { Climate } \\
\text { zone }\end{array}$ & $\begin{array}{l}\text { Source of nectar or } \\
\text { honeydew }\end{array}$ & $\begin{array}{l}\text { Pollinator/ } \\
\text { producer of } \\
\text { honeydew }\end{array}$ \\
\hline 1 & $\mathrm{fl}$ & Africa & UD & unknown & A. $m$. \\
\hline 2 & $\mathrm{fl}$ & Cuba & UD & unknown & $\begin{array}{l}\text { Melipona } \\
\text { sp. }\end{array}$ \\
\hline 3 & $\mathrm{fl}$ & Egypt & Arid & unknown & A. $m$. \\
\hline 4 & $\mathrm{fl}$ & $\begin{array}{l}\text { Hungary: Orosz- } \\
\text { lány }\end{array}$ & WSC & $\begin{array}{l}\text { Phacelia tanacetifolia } \\
\text { Benth. }\end{array}$ & A. $m$. \\
\hline 5 & $\mathrm{fl}$ & Hungary: Sárkeszi & WSC & $\begin{array}{l}\text { Foeniculum vulgare } \\
\text { Mill. }\end{array}$ & A. $m$. \\
\hline 6 & $\mathrm{fl}$ & $\begin{array}{l}\text { Hungary: Szent- } \\
\text { király }\end{array}$ & WSC & Asclepias syriaca L. & A. $m$. \\
\hline 7 & $\mathrm{fl}$ & Italy & UD & Hedysarum coronarium $\mathrm{L}$. & A. $m$. \\
\hline 8 & $\mathrm{fl}$ & Central Italy & WSC & Helianthus annuus L. & A. $m$. \\
\hline 9 & $\mathrm{fl}$ & N Italy & WSC & Castanea sativa Mill. & A. $m$. \\
\hline 10 & $\mathrm{fl}$ & N Italy & WSC & Tilia sp. & A. $m$. \\
\hline 11 & $\mathrm{fl}$ & Italy: Alps & WSC & Rhododendron sp. & A. $m$. \\
\hline 12 & $\mathrm{fl}$ & Italy: Piemonte & WSC & $\begin{array}{l}\text { Taraxacum officinale } \\
\text { Weber }\end{array}$ & A. $m$. \\
\hline 13 & $\mathrm{fl}$ & Italy: Sicily & MTN & Citrus sp. & A. $m$. \\
\hline 14 & $\mathrm{fl}$ & Mexico & UD & unknown & A. $m$. \\
\hline 15 & $\mathrm{fl}$ & New Zealand & MT & unknown & A. $m$. \\
\hline 16 & $\mathrm{fl}$ & Poland & WSC & $\begin{array}{l}\text { Fagopyrum esculentum } \\
\text { Mill. }\end{array}$ & A. $m$. \\
\hline 17 & $\mathrm{fl}$ & Portugal & MTN & Rosmarinus officinale L. & A. $m$. \\
\hline 18 & $\mathrm{fl}$ & Portugal & MTN & unknown & A. $m$. \\
\hline 19 & $\mathrm{fl}$ & South Africa & UD & Acacia sp. & A. $m$. \\
\hline 20 & $\mathrm{fl}$ & South Africa & UD & unknown & A. $m$. \\
\hline 21 & $\mathrm{fl}$ & South Africa & UD & Citrus sp. & A. $m$. \\
\hline 22 & $\mathrm{fl}$ & South Africa & UD & unknown & A. $m$. \\
\hline 23 & $\mathrm{fl}$ & South Africa & UD & unknown & A. $m$. \\
\hline 24 & $\mathrm{fl}$ & South Africa & UD & unknown & A. $m$. \\
\hline 25 & $\mathrm{fl}$ & Coastal N Spain & MT & Eucalyptus sp. & A. $m$. \\
\hline
\end{tabular}


Table 1 (continued)

\begin{tabular}{|c|c|c|c|c|c|}
\hline $\begin{array}{l}\text { Ref. } \\
\text { no. }\end{array}$ & $\begin{array}{l}\text { Honey } \\
\text { type }\end{array}$ & Locality & $\begin{array}{l}\text { Climate } \\
\text { zone }\end{array}$ & $\begin{array}{l}\text { Source of nectar or } \\
\text { honeydew }\end{array}$ & $\begin{array}{l}\text { Pollinator/ } \\
\text { producer of } \\
\text { honeydew }\end{array}$ \\
\hline 26 & $\mathrm{fl}$ & Spain & MT & Citrus sp. & A. $m$. \\
\hline 27 & $\mathrm{fl}$ & NW Spain & MT & multifloral & A. $m$. \\
\hline 28 & $\mathrm{fl}$ & NW Spain & MT & Rubus sp. & A. $m$. \\
\hline 29 & $\mathrm{fl}$ & Tanzania & UD & unknown & A. $m$. \\
\hline 30 & $\mathrm{fl}$ & Tanzania & UD & unknown & A. $m$. \\
\hline 31 & $\mathrm{fl}$ & Tunisia & MTN & unknown & A. $m$. \\
\hline 32 & hd & Bulgaria & WSC & unknown & A. $m$. \\
\hline 33 & hd & Croatia & WT & unknown & A. $m$. \\
\hline 34 & hd & Croatia & WT & unknown & A. $m$. \\
\hline 35 & hd & Greece & MTN & Abies alba Mill. & A. $m$. \\
\hline 36 & hd & Greece & MTN & Abies alba Mill. & A. $m$. \\
\hline 37 & hd & Greece & MTN & Abies alba Mill. & A. $m$. \\
\hline 38 & hd & Greece & MTN & Abies alba Mill. & A. $m$. \\
\hline 39 & hd & Greece & MTN & Abies alba Mill. & A. $m$. \\
\hline 40 & hd & Greece & MTN & Abies alba Mill. & A. $m$. \\
\hline 41 & hd & Greece & MTN & Abies alba Mill. & A. $m$. \\
\hline 42 & hd & Greece & MTN & Abies alba Mill. & A. $m$. \\
\hline 43 & hd & Greece & MTN & Abies alba Mill. & A. $m$. \\
\hline 44 & hd & Greece & MTN & Abies alba Mill. & A. $m$. \\
\hline 45 & hd & Greece & MTN & Abies alba Mill. & A. $m$. \\
\hline 46 & hd & Greece & MTN & Pinus brutia Tenore & A. $m$. \\
\hline 47 & hd & Greece & MTN & Pinus brutia Tenore & A. $m$. \\
\hline 48 & hd & Greece & MTN & Pinus brutia Tenore & A. $m$. \\
\hline 49 & hd & Greece & MTN & Pinus brutia Tenore & A. $m$. \\
\hline 50 & hd & Greece & MTN & Pinus brutia Tenore & A. $m$. \\
\hline 51 & hd & Greece & MTN & Pinus brutia Tenore & A. $m$. \\
\hline 52 & hd & Greece & MTN & Pinus brutia Tenore & A. $m$. \\
\hline 53 & hd & Greece & MTN & Pinus sp. & A. $m$. \\
\hline 54 & hd & Hungary & WSC & unknown & A. $m$. \\
\hline 55 & hd & $\begin{array}{l}\text { Hungary: JNSz } \\
\text { County }\end{array}$ & WSC & unknown & A. $m$. \\
\hline 56 & hd & Hungary: Keszthely & WSC & unknown & A. $m$. \\
\hline
\end{tabular}


Table 1 (continued)

\begin{tabular}{|c|c|c|c|c|c|}
\hline $\begin{array}{l}\text { Ref. } \\
\text { no. }\end{array}$ & $\begin{array}{l}\text { Honey } \\
\text { type }\end{array}$ & Locality & $\begin{array}{l}\text { Climate } \\
\text { zone }\end{array}$ & $\begin{array}{l}\text { Source of nectar or } \\
\text { honeydew }\end{array}$ & $\begin{array}{l}\text { Pollinator/ } \\
\text { producer of } \\
\text { honeydew }\end{array}$ \\
\hline 57 & hd & Hungary: Ózd & WSC & unknown & A. $m$. \\
\hline 58 & hd & Hungary: Sárkeszi & WSC & unknown & A. $m$. \\
\hline 59 & hd & Hungary: Solymár & WSC & unknown & A. $m$. \\
\hline 60 & hd & Italy & UD & unknown & A. $m$. \\
\hline 61 & hd & Italy & UD & unknown & M.p. + A.m. \\
\hline 62 & hd & Italy & UD & unknown & M.p. A.m. \\
\hline 63 & hd & Italy & UD & Abies alba Mill. & A. $m$. \\
\hline 64 & hd & Italy & UD & Abies alba Mill. & A. $m$. \\
\hline 65 & hd & Italy & UD & Abies alba Mill. & A. $m$. \\
\hline 66 & hd & Italy & UD & Castanea sativa Mill. & A. $m$. \\
\hline 67 & hd & Italy & UD & Castanea sativa Mill. & A. $m$. \\
\hline 68 & hd & Italy & UD & Castanea sativa Mill. & A. $m$. \\
\hline 69 & hd & Italy & UD & Castanea sativa Mill. & A. $m$. \\
\hline 70 & hd & Italy & UD & Castanea sativa Mill. & A. $m$. \\
\hline 71 & hd & Italy: Abruzzo & WT & unknown & A. $m$. \\
\hline 72 & hd & $\begin{array}{l}\text { Italy: Friuli Venezia } \\
\text { Lucia }\end{array}$ & WSC & unknown & A. $m$. \\
\hline 73 & hd & Italy: Lazio & WSC & unknown & A. $m$. \\
\hline 74 & hd & Italy: Liguria & WT & unknown & M. p. + A. m. \\
\hline 75 & hd & Italy: Liguria & WT & unknown & A. $m$. \\
\hline 76 & hd & Italy: Liguria & WT & unknown & A. $m$. \\
\hline 77 & hd & Italy: Liguria & WT & unknown & A. $m$. \\
\hline 78 & hd & Italy: Liguria & WT & unknown & M. p. +A.m. \\
\hline 79 & hd & Italy: Liguria & WT & unknown & M.p. A.m. \\
\hline 80 & hd & Italy: Liguria & WT & unknown & M.p. A.m. \\
\hline 81 & hd & Italy: Liguria & WT & unknown & A. $m$. \\
\hline 82 & hd & Italy: Liguria & WT & Abies alba Mill. & A. $m$. \\
\hline 83 & hd & Italy: Lombardia & WSC & unknown & A. $m$. \\
\hline 84 & hd & Italy: Piemonte & WSC & unknown & A. $m$. \\
\hline 85 & hd & Italy: Toscana & WSC & unknown & A. $m$. \\
\hline 86 & hd & Italy: Toscana & WSC & unknown & A. $m$. \\
\hline 87 & hd & Italy: Toscana & WSC & unknown & A. $m$. \\
\hline
\end{tabular}


Table 1 (continued)

\begin{tabular}{|c|c|c|c|c|c|}
\hline $\begin{array}{l}\text { Ref. } \\
\text { no. }\end{array}$ & $\begin{array}{l}\text { Honey } \\
\text { type }\end{array}$ & Locality & $\begin{array}{l}\text { Climate } \\
\text { zone }\end{array}$ & $\begin{array}{l}\text { Source of nectar or } \\
\text { honeydew }\end{array}$ & $\begin{array}{l}\text { Pollinator/ } \\
\text { producer of } \\
\text { honeydew }\end{array}$ \\
\hline 88 & hd & Italy: Toscana & WSC & unknown & A. $m$. \\
\hline 89 & hd & $\begin{array}{l}\text { Italy: Trentino Alto } \\
\text { Adige }\end{array}$ & WSC & unknown & A. $m$. \\
\hline 90 & hd & $\begin{array}{l}\text { Italy: Tusco-Emil- } \\
\text { ian App. }\end{array}$ & WSC & $\begin{array}{l}\text { Abies alba Mill. + Picea } \\
\text { excelsa Link }\end{array}$ & A. $m$. \\
\hline 91 & hd & Poland & WSC & unknown & A. $m$. \\
\hline 92 & hd & Slovakia & WSC & unknown & A. $m$. \\
\hline 93 & hd & Slovakia & WSC & unknown & A. $m$. \\
\hline 94 & unk & Czech Republic & WSC & unknown & A. $m$. \\
\hline 95 & unk & Czech Republic & WSC & unknown & A. $m$. \\
\hline 96 & unk & $\begin{array}{l}\text { France: Domaine } \\
\text { St. Georges }\end{array}$ & MT & unknown & A. $m$. \\
\hline 97 & unk & Germany: Altbulach & MT & unknown & A. $m$. \\
\hline 98 & unk & $\begin{array}{l}\text { Germany: Alten- } \\
\text { steig-Überberg }\end{array}$ & MT & unknown & A. $m$. \\
\hline 99 & unk & $\begin{array}{l}\text { Romania: Székely- } \\
\text { udvarhely }\end{array}$ & WSC & unknown & A. $m$. \\
\hline 100 & unk & $\begin{array}{l}\text { Switzerland: Les- } \\
\text { ponts-de-Martel }\end{array}$ & WSC & unknown & A. $m$. \\
\hline
\end{tabular}

A. m. = Apis mellifera L.

M. $p .=$ Metcalfa pruinosa Say

For each sample, $10 \mathrm{~g}$ were taken from $500 \mathrm{~g}$ of previously homogenised honey, dissolved in $20 \mathrm{ml}$ of distilled water at $40^{\circ} \mathrm{C}$, centrifuged for $10 \mathrm{~min}$ at $560 \mathrm{~g}$ and allowed to settle. The sediment was recovered in $10 \mathrm{ml}$ of distilled water and again centrifuged. The sediment was then collected with a Pasteur pipette and dried onto microscope slides at $40{ }^{\circ} \mathrm{C}$. It was then mounted in glycerine-gelatine and covered (Louveaux et al. 1978). The entire surface of each preparation $(18 \mathrm{~mm} \times 18 \mathrm{~mm})$ was scanned under $600 \times$ magnification of an Olympus CX 31 microscope. Identification of fungal spores was carried out both from experience and by means of scientific literature and monographs (Ellis and Ellis 1997, Hughes 1958, Ingold 1971, Kendrick 1990, Lacey and West 2006).

The samples were classified according to their locality into climate zones (Biondi and Baldoni 1994, Peel et al. 2007). If the geographical source of the honey was not known specifically enough, e.g. in the case of certain honeys 
from Italy, the sample's climate zone was described as "undefined", because more than one zone is covered by the respective country.

Non-parametric comparative methods were used to test the difference between the spore type diversities in floral and honeydew honeys. Kruskal-Wallis rank sum tests were performed to see whether the botanical source or the climate of the locality affects this kind of diversity. The statistical tests were carried out in R v.3.0.2. Diagrams were generated in Microsoft Office Excel 2010.

\section{RESULTS}

Diversity of fungal taxa in samples

A total of 227 types of spores (Table 2) were found in 100 samples. We identified 94 of them on genus level, while 81 were specified on species level. The following types were present in more than half of all of our honey

\section{Table 2}

List of recorded spore types and the percentage of honeydew honey (HD) and floral honey (FL) samples where they were recorded

\begin{tabular}{clcc}
\hline $\begin{array}{c}\text { Ref. } \\
\text { no. }\end{array}$ & Spore type & $\begin{array}{c}\text { HD } \\
(\%)\end{array}$ & $\begin{array}{c}\text { FL } \\
(\%)\end{array}$ \\
\hline 1 & Acroconidiella tropaeoli (T. E. T. Bond) J. C. Lindq. et Alippi & 0.00 & 12.90 \\
2 & Acrodictys sp. & 6.45 & 6.45 \\
3 & Agaricomycetes & 61.29 & 38.71 \\
4 & Aglaospora profusa (Fr.) De Not. & 11.29 & 3.23 \\
5 & Agrocybe sp. & 12.90 & 6.45 \\
6 & Albugo sp. & 12.90 & 3.23 \\
7 & Alternaria ? alternata (Fr.) Keissl. & 9.68 & 3.23 \\
8 & Alternaria sp. & 90.32 & 80.65 \\
9 & Ampulliferina persimplex B. Sutton & 1.61 & 0.00 \\
10 & Anellophora sp. ? & 16.13 & 3.23 \\
11 & Antennatula sp. & 14.52 & 3.23 \\
12 & Anthostomella/Herpotrichiella spp. & 27.42 & 0.00 \\
13 & Anthracoidea sp. & 8.06 & 0.00 \\
14 & Arthrinium cuspidatum (Cooke et Harkn.) Tranzschel & 1.61 & 3.23 \\
15 & Arthrinium sp. & 43.55 & 12.90 \\
16 & Articulospora sp. & 3.23 & 0.00 \\
17 & Ascobolus sp. & 4.84 & 0.00 \\
18 & Aspergillus/Penicillium spp. & 4.84 & 12.90 \\
\hline & & &
\end{tabular}


Table 2 (continued)

\begin{tabular}{|c|c|c|c|}
\hline $\begin{array}{c}\text { Ref. } \\
\text { no. }\end{array}$ & Spore type & $\begin{array}{l}\text { HD } \\
(\%)\end{array}$ & $\begin{array}{l}\text { FL } \\
(\%) \\
\end{array}$ \\
\hline 19 & Aspergillus sect. Nigri & 1.61 & 0.00 \\
\hline 20 & Asterosporium asterospermum (Pers.) S. Hughes & 1.61 & 0.00 \\
\hline 21 & Atichia millardetii Racib. & 22.58 & 3.23 \\
\hline 22 & Aureobasidium pullulans (de Bary et Löwenthal) G. Arnaud & 25.81 & 3.23 \\
\hline 23 & Belemnospora verruculosa P. M. Kirk & 16.13 & 9.68 \\
\hline 24 & Beltrania rhombica Penz. & 1.61 & 3.23 \\
\hline 25 & Bipolaris sp. & 11.29 & 0.00 \\
\hline 26 & Bipolaris spicifera (Bainier) Subram. & 14.52 & 6.45 \\
\hline 27 & Boletaceae & 16.13 & 6.45 \\
\hline 28 & Botrytis sp. & 30.65 & 6.45 \\
\hline 29 & Bovista sp. & 1.61 & 0.00 \\
\hline 30 & Caloplaca sp. & 29.03 & 0.00 \\
\hline 31 & Camarosporium sp. & 14.52 & 3.23 \\
\hline 32 & Camposporium sp. & 1.61 & 0.00 \\
\hline 33 & Capnobotrys sp. & 16.13 & 3.23 \\
\hline 34 & Ceratosporium cornutum Matsush. & 8.06 & 0.00 \\
\hline 35 & Cercospora sp. & 4.84 & 9.68 \\
\hline 36 & Cerebella andropogonis Ces. & 0.00 & 16.13 \\
\hline 37 & Chaetomium sp. & 14.52 & 9.68 \\
\hline 38 & Chaetosphaerella sp. & 9.68 & 0.00 \\
\hline 39 & Cheiromycella microscopica (P. Karst.) S. Hughes & 24.19 & 0.00 \\
\hline 40 & Cladosporium ? aecidiicola Thüm. & 1.61 & 0.00 \\
\hline 41 & Cladosporium ? phlei (C. T. Greg.) G. A. de Vries & 25.81 & 3.23 \\
\hline 42 & Cladosporium spp. & 69.35 & 48.39 \\
\hline 43 & Clasterosporium sp.? & 37.10 & 3.23 \\
\hline 44 & Clypeosphaeria notarisii Fuckel & 32.26 & 0.00 \\
\hline 45 & Colletotrichum sp. & 4.84 & 0.00 \\
\hline 46 & Coniosporium sp. & 11.29 & 3.23 \\
\hline 47 & Coprinus? niveus (Pers.) Fr. & 4.84 & 0.00 \\
\hline 48 & Coprinus sp. & 37.10 & 22.58 \\
\hline 49 & Cortinarius sp. & 22.58 & 3.23 \\
\hline 50 & Corynespora spp.? & 9.68 & 6.45 \\
\hline 51 & Coryneum sp. & 14.52 & 3.23 \\
\hline
\end{tabular}


Table 2 (continued)

\begin{tabular}{|c|c|c|c|}
\hline $\begin{array}{l}\text { Ref. } \\
\text { no. }\end{array}$ & Spore type & $\begin{array}{l}\text { HD } \\
(\%)\end{array}$ & $\begin{array}{l}\text { FL } \\
(\%)\end{array}$ \\
\hline 52 & Cucurbitaria sp. & 6.45 & 0.00 \\
\hline 53 & Curucispora sp.* & 0.00 & 0.00 \\
\hline 54 & Curvularia brachyspora Boedijn & 0.00 & 3.23 \\
\hline 55 & Curvularia catenulata S. M. Reddy et Bilgrami & 0.00 & 3.23 \\
\hline 56 & Curvularia geniculata (Tracy et Earle) Boedijn & 0.00 & 3.23 \\
\hline 57 & Curvularia leonensis M. B. Ellis & 0.00 & 3.23 \\
\hline 58 & Curvularia spp. & 9.68 & 25.81 \\
\hline 59 & Dendryphion digitatum Subram. & 12.90 & 0.00 \\
\hline 60 & Diapleella clivensis? (Berk. et Broome) Munk. & 11.29 & 0.00 \\
\hline 61 & Diatrypaceae & 0.00 & 3.23 \\
\hline 62 & Dicranidion sp. & 11.29 & 0.00 \\
\hline 63 & Dictyopolyschema pirozynskii M. B. Ellis & 17.74 & 0.00 \\
\hline 64 & Dictyosporium toruloides (Corda) Guég. & 1.61 & 6.45 \\
\hline 65 & Diplocladiella scalaroides G. Arnaud ex M. B. Ellis & 1.61 & 3.23 \\
\hline 66 & $\begin{array}{l}\text { Diplodia frumenti Ellis et Everh./ Lasiodiplodia theobromae } \\
\text { (Pat.) Griffon et Maubl. }\end{array}$ & 0.00 & 16.13 \\
\hline 67 & Diplodia spp. & 30.65 & 9.68 \\
\hline 68 & Discostroma corticola (Fuckel) Brockmann & 37.10 & 6.45 \\
\hline 69 & $\begin{array}{l}\text { Drechslera biseptata (Sacc. et Roum.) M. J. Richardson et E. M. } \\
\text { Fraser }\end{array}$ & 3.23 & 3.23 \\
\hline 70 & Dwayaangam dichotoma Nawawi & 1.61 & 0.00 \\
\hline 71 & Dwayangaam spp. & 1.61 & 0.00 \\
\hline 72 & Endophragmia bisby B. Sutton & 4.84 & 0.00 \\
\hline 73 & Endophragmiella taxi (M. B. Ellis) S. Hughes & 1.61 & 0.00 \\
\hline 74 & Epicoccum nigrum Link & 82.26 & 77.42 \\
\hline 75 & Excipularia fusispora (Berk. et Broome) Sacc. & 45.16 & 6.45 \\
\hline 76 & Excipularia narsapurensis Subram. & 0.00 & 3.23 \\
\hline 77 & Exserohilum sp. & 3.23 & 3.23 \\
\hline 78 & Flabellospora sp. & 4.84 & 3.23 \\
\hline 79 & Fusarium spp. (macroconidia) & 16.13 & 6.45 \\
\hline 80 & Fusicladium sp. & 9.68 & 0.00 \\
\hline 81 & Ganoderma sp. & 58.06 & 6.45 \\
\hline 82 & Gyoerffyella myrmecophagiformis Melnik and Dudka & 1.61 & 0.00 \\
\hline
\end{tabular}


Table 2 (continued)

\begin{tabular}{|c|c|c|c|}
\hline $\begin{array}{l}\text { Ref. } \\
\text { no. }\end{array}$ & Spore type & $\begin{array}{l}\text { HD } \\
(\%)\end{array}$ & $\begin{array}{l}\text { FL } \\
(\%) \\
\end{array}$ \\
\hline 83 & Helicogermslita sp. & 0.00 & 3.23 \\
\hline 84 & Helicosporium sp. & 12.90 & 3.23 \\
\hline 85 & $\begin{array}{l}\text { Helicosporium state of Tubeufia palmarum (Torrend) Samuels, } \\
\text { Rossman et E. Müll.* }\end{array}$ & 0.00 & 0.00 \\
\hline 86 & Helminthosporium/Drechslera spp. & 48.39 & 32.26 \\
\hline 87 & Hypoxylon fuscum? (Pers.) Fr. & 17.74 & 3.23 \\
\hline 88 & Isthmolongispora ampulliformis (Tubaki) de Hoog et Hennebert & 1.61 & 0.00 \\
\hline 89 & Isthmospora spinosa F. L. Stevens & 1.61 & 0.00 \\
\hline 90 & Isthmotricladia sp. & 8.06 & 0.00 \\
\hline 91 & Lactarius/Russula sp. & 1.61 & 0.00 \\
\hline 92 & Lasiosphaeria spp. & 3.23 & 0.00 \\
\hline 93 & Lemonniera sp. & 8.06 & 3.23 \\
\hline 94 & Leptosphaeria pleurospora Niessl & 1.61 & 0.00 \\
\hline 95 & Leptosphaeria thurgoviensis E. Müll. & 1.61 & 0.00 \\
\hline 96 & Leptosphaeria spp. & 50.00 & 19.35 \\
\hline 97 & Lophiostoma vicinum Sacc. & 27.42 & 6.45 \\
\hline 98 & Lylea tetracoila (Corda) Hol.-Jech. & 1.61 & 00.00 \\
\hline 99 & Melampsoridium/Cronartium/Melampsora spp. & 62.90 & 22.58 \\
\hline 100 & Metschnikowia reukaufii Pitt. et M. W. Miller & 27.42 & 58.06 \\
\hline 101 & Microbotryum reticulatum (Liro) R. Bauer et Oberw. & 8.06 & 0.00 \\
\hline 102 & Microbotryum violaceum (Pers.) G. Deml et Oberw. & 14.52 & 3.23 \\
\hline 103 & Mucorales & 1.61 & 0.00 \\
\hline 104 & Mycocentrospora sp. & 3.23 & 0.00 \\
\hline 105 & Mycosphaerella sp. & 1.61 & 0.00 \\
\hline 106 & Myxomycetes & 56.45 & 29.03 \\
\hline 107 & Nakataea sigmoidea (Cavara) Hara & 14.52 & 3.23 \\
\hline 108 & Neohendersonia kickxii (Westend.) B. Sutton et Pollack & 9.68 & 0.00 \\
\hline 109 & Nigrospora sp. & 11.29 & 19.35 \\
\hline 110 & Oidium sp. & 20.97 & 3.23 \\
\hline 111 & Oncopodiella trigonella (Sacc.) Rifai & 6.45 & 0.00 \\
\hline 112 & Ovulariopsis sp. & 1.61 & 0.00 \\
\hline 113 & Paraphaeosphaeria michotii (Westend.) O. E. Erikss. & 17.74 & 3.23 \\
\hline 114 & Parapyricularia sp.? & 8.06 & 0.00 \\
\hline
\end{tabular}


Table 2 (continued)

\begin{tabular}{|c|c|c|c|}
\hline $\begin{array}{c}\text { Ref. } \\
\text { no. }\end{array}$ & Spore type & $\begin{array}{l}\mathrm{HD} \\
(\%)\end{array}$ & $\begin{array}{l}\text { FL } \\
(\%)\end{array}$ \\
\hline 115 & Passalora sp. & 20.97 & 0.00 \\
\hline 116 & Periconia sp. & 61.29 & 35.48 \\
\hline 117 & Periconia/Tilletia sp.* & 0.00 & 0.00 \\
\hline 118 & Peronosporaceae-type sporangia & 22.58 & 9.68 \\
\hline 119 & Pestalotiopsis spp. & 16.13 & 9.68 \\
\hline 120 & Pestalotiopsis stevensonii (Peck) Nag Raj & 11.29 & 0.00 \\
\hline 121 & Phragmidium sp. & 3.23 & 0.00 \\
\hline 122 & Pithomyces? cynodontis M. B. Ellis & 1.61 & 6.45 \\
\hline 123 & Pithomyces chartarum (Berk. et M. A. Curtis) M. B. Ellis & 50.00 & 25.81 \\
\hline 124 & Pleospora rubelloides (Plowr. ex Cooke) J. Webster & 14.52 & 0.00 \\
\hline 125 & Pleospora sp. & 33.87 & 12.90 \\
\hline 126 & Pollaccia elegans Servazzi & 1.61 & 0.00 \\
\hline 127 & Pollaccia sp. & 6.45 & 0.00 \\
\hline 128 & Polythrincium trifolii Speg. & 45.16 & 6.45 \\
\hline 129 & Psammina sp. & 0.00 & 0.00 \\
\hline 130 & Pucciniaceae spp. (teliospores) & 3.23 & 0.00 \\
\hline 131 & Pucciniaceae spp. (uredospores) & 59.68 & 48.39 \\
\hline 132 & Pyrigemmula aurantiaca D. Magyar et Shoemaker & 22.58 & 0.00 \\
\hline 133 & Rebentischia unicaudata (Berk. et Broome) Sacc. & 16.13 & 0.00 \\
\hline 134 & Retiarius bovicornutus D. L. Olivier & 12.90 & 0.00 \\
\hline 135 & Retiarius/Trinacrium spp. & 8.06 & 0.00 \\
\hline 136 & Rhizopus sp. & 0.00 & 3.23 \\
\hline 137 & Rhynchosporium sp. & 1.61 & 0.00 \\
\hline 138 & Sarcostroma arbuti (Bonar) M. Morelet & 3.23 & 0.00 \\
\hline 139 & Scopinella sp. & 1.61 & 0.00 \\
\hline 140 & Scopulariopsis sp. & 1.61 & 0.00 \\
\hline 141 & Seimatosporium sp. & 11.29 & 0.00 \\
\hline 142 & Sirosporium/Acrodictys sp. & 8.06 & 0.00 \\
\hline 143 & Sordaria sp. & 1.61 & 0.00 \\
\hline 144 & Spegazzinia sp. & 4.84 & 29.03 \\
\hline 145 & Sphaeropsis sp. 1 & 9.68 & 0.00 \\
\hline 146 & Sphaeropsis sp. 2 & 1.61 & 0.00 \\
\hline 147 & Spilocaea spp. & 37.10 & 6.45 \\
\hline
\end{tabular}


Table 2 (continued)

\begin{tabular}{|c|c|c|c|}
\hline $\begin{array}{l}\text { Ref. } \\
\text { no. }\end{array}$ & Spore type & $\begin{array}{l}\text { HD } \\
(\%)\end{array}$ & $\begin{array}{l}\text { FL } \\
(\%) \\
\end{array}$ \\
\hline 148 & Splanchnonema spp. & 4.84 & 0.00 \\
\hline 149 & Sporidesmium brachypus (Ellis et Everh.) S. Hughes & 4.84 & 0.00 \\
\hline 150 & Sporidesmiella brachysporioides T. Y. Zhang et W. B. Kendr.* & 0.00 & 0.00 \\
\hline 151 & Sporidesmiella hyalosperma (Corda) P. M. Kirk & 6.45 & 0.00 \\
\hline 152 & Sporidesmium leptosporum (Sacc. et Roum.) S. Hughes & 1.61 & 0.00 \\
\hline 153 & Sporidesmium macrotrichum (Corda) S. Hughes & 8.06 & 0.00 \\
\hline 154 & Sporidesmium spp. & 25.81 & 3.23 \\
\hline 155 & Sporormiella sp. & 8.06 & 3.23 \\
\hline 156 & Stachybotrys sp. & 19.35 & 3.23 \\
\hline 157 & Stegonsporium sp. & 1.61 & 0.00 \\
\hline 158 & Stemphylium solani G. F. Weber & 3.23 & 0.00 \\
\hline 159 & Stemphylium spp. & 67.74 & 41.94 \\
\hline 160 & Stenellopsis fagraeae B. Huguenin & 1.61 & 0.00 \\
\hline 161 & Stigmina sp. & 1.61 & 0.00 \\
\hline 162 & Taeniolella ? breviuscula (Berk. et M. A. Curtis) S. Hughes & 3.23 & 0.00 \\
\hline 163 & Taeniolella sp. & 48.39 & 6.45 \\
\hline 164 & Telephoraceae & 14.52 & 3.23 \\
\hline 165 & Teloschistes/Xanthoria sp. & 1.61 & 3.23 \\
\hline 166 & Tetraploa aristata Berk. et Broome & 1.61 & 3.23 \\
\hline 167 & Thielaviopsis sp. & 1.61 & 0.00 \\
\hline 168 & Thyrostroma negundinis (Berk. et M. A. Curtis) A. W. Ramaley & 4.84 & 0.00 \\
\hline 169 & Tilletia sp. & 9.68 & 0.00 \\
\hline 170 & Torula sp. & 48.39 & 54.84 \\
\hline 171 & Toxosporium sp. & 0.00 & 0.00 \\
\hline 172 & Tranzscheliella hypodytes (Schltdl.) Vánky et McKenzie & 11.29 & 3.23 \\
\hline 173 & Tranzscheliella williamsii (Griffiths) Dingley et Versluys & 8.06 & 3.23 \\
\hline 174 & Triadelphia heterospora Shearer et J. L. Crane & 9.68 & 0.00 \\
\hline 175 & Triadelphia uniseptata (Berk. et Broom) P. M. Kirk & 19.35 & 3.23 \\
\hline 176 & Tricellula sp. & 4.84 & 0.00 \\
\hline 177 & Trichotecium roseum (Pers.) Link & 1.61 & 3.23 \\
\hline 178 & Tricladium angulatum Ingold $^{*}$ & 0.00 & 0.00 \\
\hline 179 & Trifurcospora sp. & 4.84 & 0.00 \\
\hline 180 & Trimmatostroma salicis Corda & 25.81 & 0.00 \\
\hline
\end{tabular}


Table 2 (continued)

\begin{tabular}{|c|c|c|c|}
\hline $\begin{array}{c}\text { Ref. } \\
\text { no. }\end{array}$ & Spore type & $\begin{array}{l}\text { HD } \\
(\%)\end{array}$ & $\begin{array}{l}\text { FL } \\
(\%)\end{array}$ \\
\hline 181 & Trimmatostroma scutellare (Berk. et Broome) M. B. Ellis & 11.29 & 0.00 \\
\hline 182 & Trinacrium gracile Matsush. & 1.61 & 0.00 \\
\hline 183 & Trinacrium incurvum Matsush. & 1.61 & 0.00 \\
\hline 184 & Trinacrium robustum Tzean et J. L. Chen & 1.61 & 0.00 \\
\hline 185 & Trinacrium sp. 1 & 11.29 & 0.00 \\
\hline 186 & Trinacrium sp. 2 & 1.61 & 0.00 \\
\hline 187 & Trinacrium subtile Riess & 3.23 & 0.00 \\
\hline 188 & Tripospermum camelopardus Ingold, Dann et P. J. McDougall & 16.13 & 0.00 \\
\hline 189 & Tripospermum spp. & 70.97 & 12.90 \\
\hline 190 & Urocystis sp. & 8.06 & 0.00 \\
\hline 191 & Ustilago bromivora (Tul. et C. Tul.) A. A. Fisch. Waldh. & 24.19 & 12.90 \\
\hline 192 & Ustilago sp. & 43.55 & 29.03 \\
\hline 193 & Valsaria ? insitiva ( Tode) Ces. et De Not. & 9.68 & 0.00 \\
\hline 194 & Varicosporium elodeae W. Kegel & 8.06 & 0.00 \\
\hline 195 & Xylariaceae & 45.16 & 12.90 \\
\hline 196 & Zygophiala jamaicensis E. W. Mason. & 14.52 & 6.45 \\
\hline 197 & Zygosporium geminatum S. Hughes & 0.00 & 3.23 \\
\hline 198 & Zygosporium masonii S. Hughes? & 1.61 & 0.00 \\
\hline 199 & unknown Ascomycetes (Nectria?) & 3.23 & 0.00 \\
\hline 200 & unknown Ascomycetes (Venturia?) & 6.45 & 0.00 \\
\hline 201 & unknown helicospore type 1 & 3.23 & 0.00 \\
\hline 202 & unknown phaeoamerospore type 1 (Mammaria?) & 19.35 & 0.00 \\
\hline 203 & unknown phaeoamerospore type 2 & 11.29 & 0.00 \\
\hline 204 & unknown phaeodidymospore type 2 & 4.84 & 0.00 \\
\hline 205 & unknown phaeophragmospore type 1 & 1.61 & 0.00 \\
\hline 206 & unknown cheirospore type 1 & 0.00 & 0.00 \\
\hline 207 & unknown scolecospore type 1 & 33.87 & 0.00 \\
\hline 208 & unknown scolecospore type 2 & 4.84 & 3.23 \\
\hline 209 & unknown scolecospore type 3 & 16.13 & 0.00 \\
\hline 210 & unknown scolecospore type 4 & 3.23 & 0.00 \\
\hline 211 & unknown scolecospore type 5 & 1.61 & 0.00 \\
\hline 212 & unknown scolecospore type 6 & 8.06 & 0.00 \\
\hline 213 & unknown scolecospore type 7 & 1.61 & 0.00 \\
\hline
\end{tabular}


Table 2 (continued)

\begin{tabular}{llcc}
\hline $\begin{array}{r}\text { Ref. } \\
\text { no. }\end{array}$ & Spore type & $\begin{array}{c}\text { HD } \\
(\%)\end{array}$ & $\begin{array}{c}\text { FL } \\
(\%)\end{array}$ \\
\hline 214 & unknown Atichia-like spore type & 53.23 & 3.23 \\
215 & unknown staurospore type 1 & 3.23 & 0.00 \\
216 & unknown staurospore type 3 & 1.61 & 3.23 \\
217 & unknown staurospore type 4 & 1.61 & 0.00 \\
218 & unknown staurospore type 5 & 1.61 & 0.00 \\
219 & unknown staurospore type 6 & 4.84 & 0.00 \\
220 & unknown staurospore type 7 & 1.61 & 0.00 \\
221 & unknown staurospore type 8 & 1.61 & 0.00 \\
222 & unknown staurospore type 9 & 1.61 & 0.00 \\
223 & unknown staurospore type 10 & 1.61 & 0.00 \\
224 & unknown staurospore type 11* & 0.00 & 0.00 \\
225 & unknown staurospore type 12* & 0.00 & 0.00 \\
226 & unknown staurospore type 13* & 0.00 & 0.00 \\
227 & unknown staurospore type 14* & 0.00 & 0.00 \\
\hline
\end{tabular}

*Spore type that only occurred in samples without pre-specified botanical origin (not classified as honeydew or floral honey)

samples: Alternaria (88\%), Epicoccum (81\%), Cladosporium (65\%), Stemphylium (59\%), Pucciniaceae uredospores (57\%), miscellaneous Agaricomycetes basidiospores (55\%). Tripospermum occurred in 53\% of samples. Precisely half of the honey samples contained spores belonging to Melampsoridium/Cronartium/Melampsora group, Torula spp. and Aspergillus/Penicillium type conidia.

In this study, means of the number of identified spore types differed between honeydew $(30.27 \pm 15.21)$ and floral honeys (11.87 \pm 5.64$)$. Non-parametric methods showed significant difference between these two main groups of samples $\left(\mathrm{p}=2.002 \times 10^{-16}\right.$ for Kolmogorov-Smirnov test, $\mathrm{p}=1.301 \times 10^{-13}$ for Brunnel-Munzel test). The mean of the number of spore types $(46.20 \pm 12.46)$ was outstanding in the case of Castanea sativa honeys $(\mathrm{N}=5)$. Abies $(\mathrm{N}=16)$ samples had a mean of $27.94 \pm 10.74$ while in honeydew honeys from Pinus trees $(\mathrm{N}=8)$ averagely $14.75 \pm 9.02$ types of spores were present (Fig. 2).

Honeys from the maritime temperate zone $(\mathrm{N}=8,17.00 \pm 12.40)$ and from the Mediterranean regions $(\mathrm{N}=23,18.17 \pm 9.25)$ contained averagely a lower number of spore types than honeys from other categories (Fig. 3). Samples from warm temperate regions $(\mathrm{N}=12)$ had the highest mean $(34.25 \pm 15.88)$. At the same time, honeys coming from the warm summer continental zone $(\mathrm{N}=$ $33,24.44 \pm 15.18$ ) reached an intermediate value. 
Based on Kruskal-Wallis rank sum tests, differences in the number of spore types found are statistically significant regarding the botanical source $\left(\mathrm{p}=1.29 \times 10^{-9}\right)$ and the climatic categories $\left(\mathrm{p}=2.28 \times 10^{-2}\right)$ as well.

\section{Comparison between the most occurring taxa in honeydew and floral honeys}

The 13 most frequent taxa of honeydew honeys and floral honeys were also compared (Figs 4-5). The genera Alternaria and Epicoccum were the most encountered in both main kinds of honey. However, they were found in a higher percentage of honeydew honeys, as the ratio of Alternaria-containing samples was $9.68 \%$ higher and the ratio of Epicoccum-containing honeys was $4.84 \%$ higher among honeydew honeys. The third most frequent taxa were, however, different. Metschnikowia reukaufii was found in $58.06 \%$ of all floral honey samples, while Tripospermum spp. were present in $70.97 \%$ of honeydew honeys.

Although Cladosporium genus was found in $69.35 \%$ of honeydew honeys, only $48.39 \%$ of floral honeys contained its spores. Uredospores of family Pucciniaceae were just as frequently encountered in floral honeys as conidia of Cladosporium, but in honeydew honeys, they are ranked ninth with a percentage of $59.67 \%$. Melampsoridium/Cronartium/Melampsora spp. spores that were counted separately from those of other rust fungi were among the most frequently encountered in honeydew honeys with $62.90 \%$, but they were not even in the first ten most occurring taxa of floral honeys.

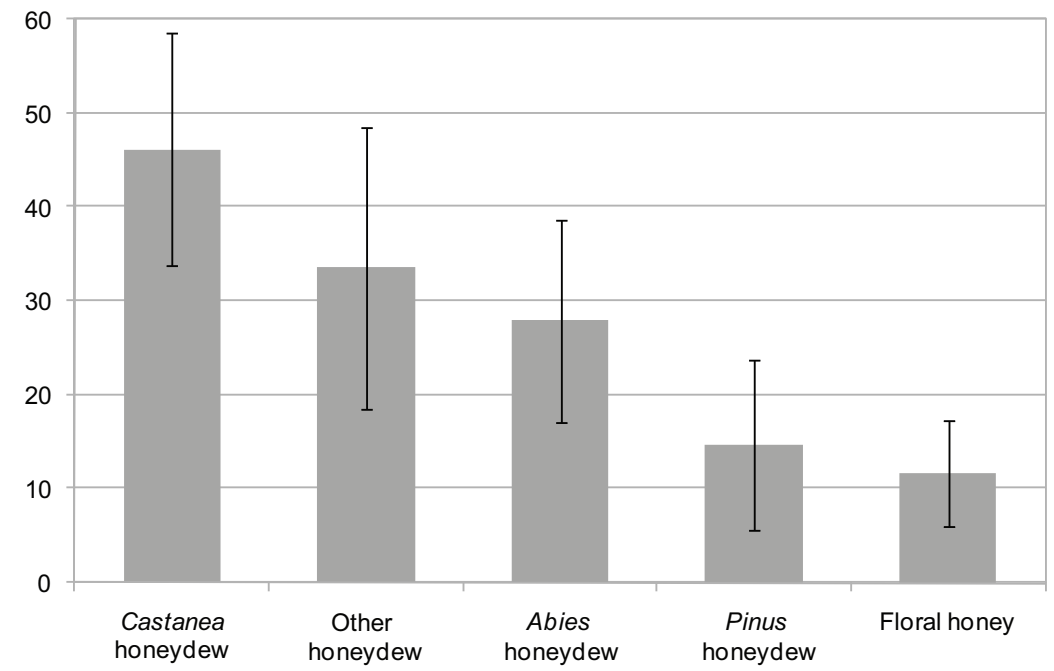

Fig. 2. The means of the number of spore types according to the botanical source of the honeys 


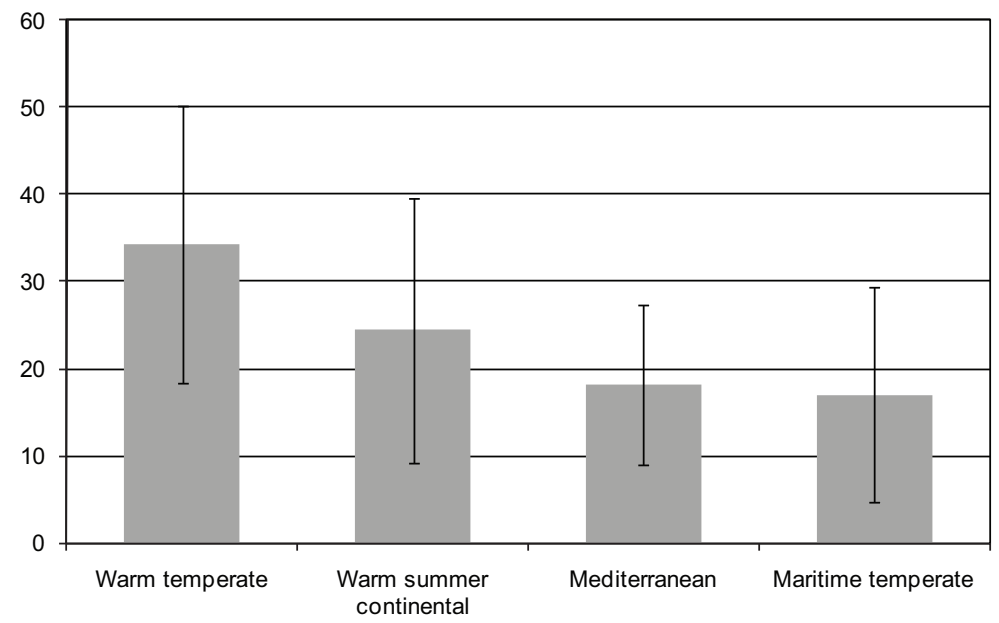

Fig. 3. The means of the number of spore types according to the climate zones of the source locations

The median of the first thirteen percentages in honeydew honeys is $61.29 \%$ that corresponds to Periconia and to the category of miscellaneous Agaricomycetes. The median is $41.94 \%$ in the case of floral honeys, belonging to Stemphylium. Myxomycetes, Ustilago and Spegazzinia were found in $29.03 \%$ of floral honeys, while Myxomycetes were present in $56.45 \%$ of honeydew hon-

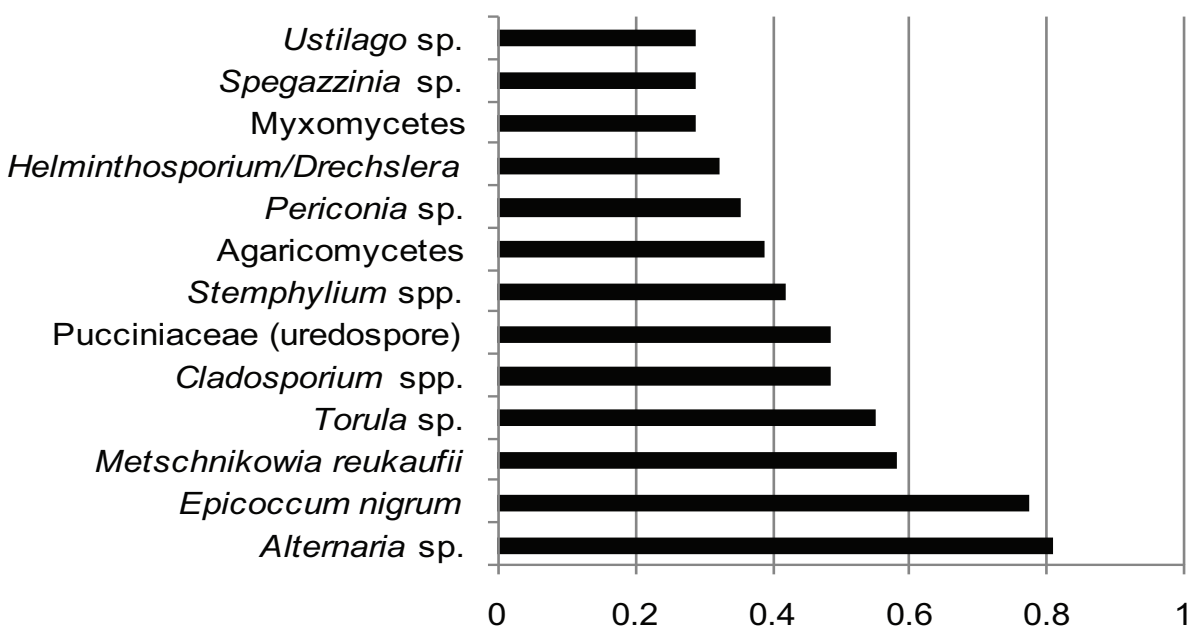

Fig. 4. The 13 most frequent spore types found in floral honeys. The scale corresponds to the ratio of honey samples, which contained the respective spore type 


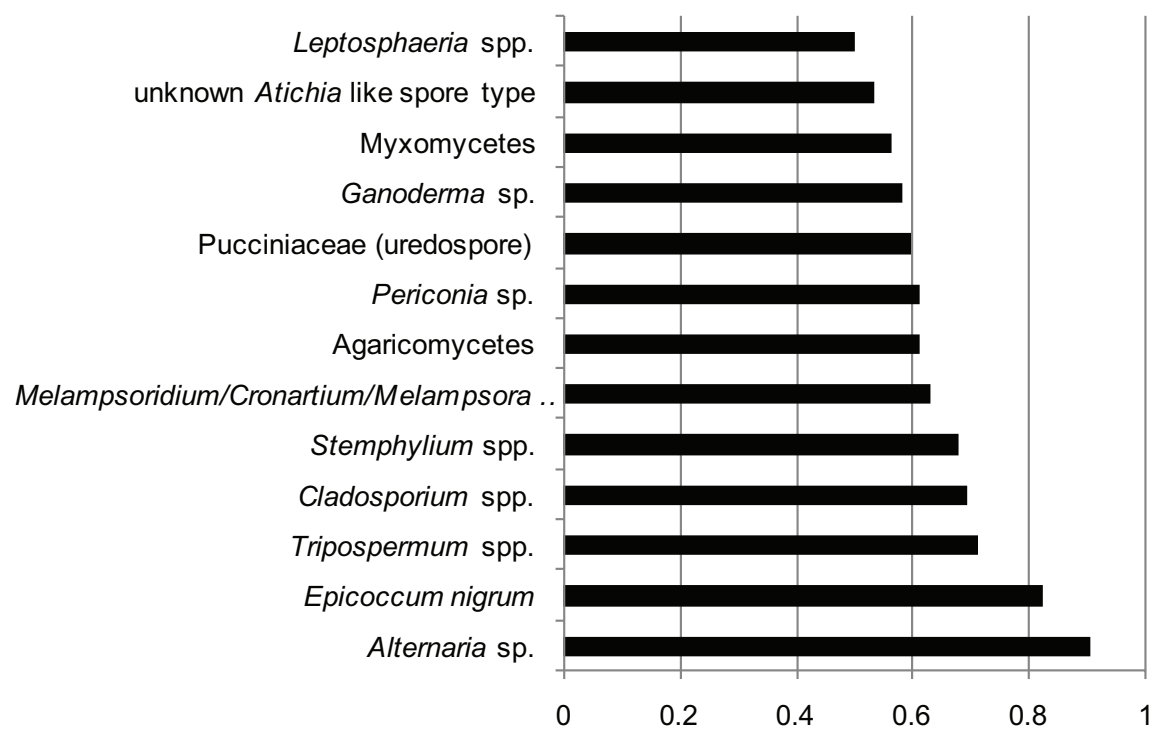

Fig. 5. The 13 most frequent spore types found in honeydew honeys. The scale corresponds to the ratio of honey samples, which contained the respective spore type

eys. Ustilago and Spegazzinia were encountered in less than $50 \%$ of non-floral honey samples. In honeydew honeys, Leptosphaeria was the thirteenth most occurring with 50\%. Between Myxomycetes and Leptosphaeria, an unknown Atichia-like spore type is also ranked with $53.23 \%$ in honeydew honeys.

Honey samples, especially those of honeydew origin had rich content of stauroid spores. Examples of the stauroid fungal spores found are shown in Figure 6.

\section{DISCUSSION}

Hereby, we attempted to investigate the widest possible spectrum of fungal spores in a hundred of honey samples collected from diverse geographical localities. Our results suggest that the most frequent fungal taxa in floral and honeydew honeys belong to ubiquitous species of fungi (Alternaria, Cladosporium, Epicoccum, Stemphylium). Therefore, it seems probable that these types of spores come from various sources and are not typical of the botanical or geographical origin of honey. On the other hand, less frequent fungi are apparently more specific. According to previous findings, floral honeys contained nectar-inhabiting Metschnikowia cells (Magyar et al. 2005, Seijo et al. 2011). The fungi found in honeydew honey samples represent phyllosphere fungi, including sooty moulds, and fungi with airborne or unknown origin. Sooty 


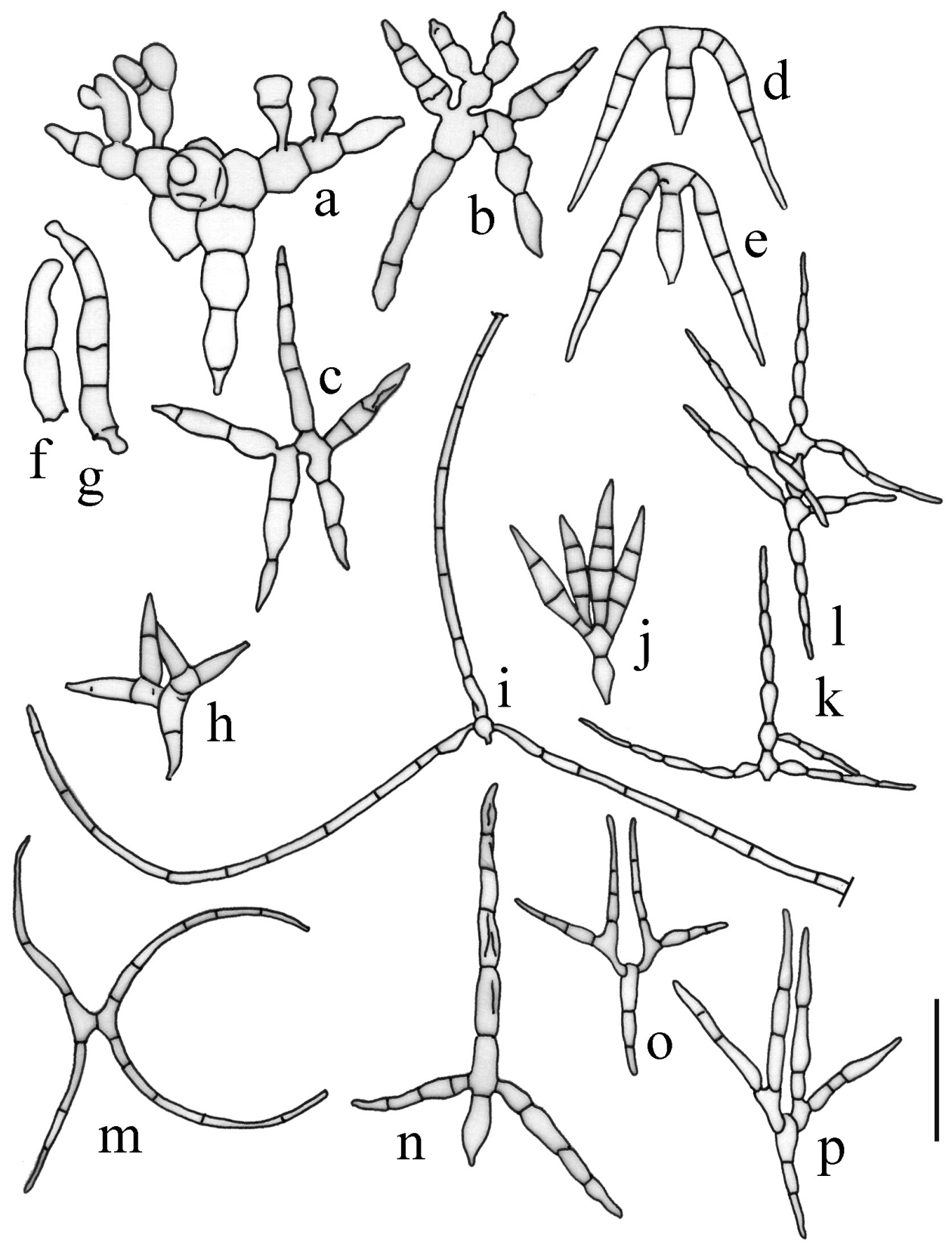

Fig. 6. Some of the fungal spores detected in honeys: $\mathrm{a}-\mathrm{c}=$ Tripospermum spp., $\mathrm{d}-\mathrm{e}=$ Trinacrium sp. 1, $\mathrm{f}-\mathrm{g}=$ unknown scolecospore type $7, \mathrm{~h}=$ unknown staurospore type $9, \mathrm{i}=$ unknown staurospore type $13, \mathrm{j}=$ Isthmotricladia/Tridentaria sp., $\mathrm{k}-\mathrm{l}=$ unknown staurospore type 11 , $\mathrm{m}=$ Curucispora sp., $\mathrm{n}=$ unknown staurospore type $10, \mathrm{o}-\mathrm{p}=$ Dwayaangam $\mathrm{sp}$. Bar $=20 \mu \mathrm{m}$ 
moulds are a heterogeneous group including Antennatula, Aureobasidium, Cladosporium, and Tripospermum as well. Most of them are primarily growing on plant surfaces covered with honeydew that is secreted by sucking-piercing insects, e.g. aphids. These fungi are characterised by dark coloured hyphae and spores, because their pigmentation becomes intense due to exposure to sunlight. Other phyllosphere fungi belong to two major groups, namely to plant pathogens and to a group of saprotrophs not dependent on the presence of honeydew (e.g. lignin and hemicellulose decomposers). Some of them are more or less host specific (e.g. Pollaccia elegans on Populus spp.) and because of this, the presence of their spores in honeydew honey may be worthy to note, when the aim is to determine the botanical origin of the sample.

Other phyllosphere fungi may indicate honeydew origin from woody plants. For instance bark inhabiting fungi (Excipularia, Oncopodiella, Pyrigemmula spp.) might be more abundant in these samples. Such fungi are living on the bole, the branches and on the twigs of the trees, but not only at the bark surface. A hidden habitat of less known groups of fungi was found under the bark surface (between horizontal layers of the bark, Magyar 2008, Magyar and Révay 2009, Magyar et al. 2011). Underbark fungi are transported to and from the surface via cracks and fissures on the bark (Magyar 2008). Some fungi found in honeys are considered to be non-specific to the host, being common saprotrophs, e.g. Alternaria and Cladosporium. They are not only common on honeydew, but on leaf litter, decaying plant materials as well. The majority of the above mentioned fungi could be trapped in the honeydew by being in contact with the fungal colony. Spontaneous spores (originated from sources other than the host plant or from contamination during harvest and processing) could also be detected in honey samples, and their non-specific nature becomes evident when identified (e.g. basidiospores of Cortinarius, or the spores of coprophilous fungi, e.g. Ascobolus).

Identification of fungi in honey samples could therefore be a useful tool when it serves the purpose to determine the botanical origin of the honey or to prove its originality. Although the qualitative (presence or absence) data of fungi could be used for that sake, the quantitative data may be even more useful, and merit further studies. More research is therefore needed to calculate the indicator value of these fungi.

Acknowledgements - The authors are grateful to Dr László Békési and Dr Zsuzsanna Szél (Institute of Apiculture, Gödöllő, Hungary) for providing additional samples for microscopical examination. The authors thank Dr Szilvia Barna, Ágnes Schütz and Orsolya Tekes (National Public Health Centre, Budapest, Hungary) for their help in the preparation of slides. We also thank Dr Irén Siller (Faculty of Veterinary Science, Szent István University, Budapest, Hungary) for her advice concerning the present study. 


\section{REFERENCES}

Biondi, E. and Baldoni, M. (1994): Climate and vegetation of the Italian peninsula. - Anais Inst. Super. Agron. 44: 75-135.

Bogdanov, S. and Martin, P. (2002): Honey authenticity: a review. - Mitt. Lebensmitt. Hyg. 93: 232-254.

Dimou, M., Katsaros, J., Klonari, K. T. and Thrasyvoulou, A. (2006): Discriminating pine and fir honeydew honey by microscopic characteristics. - J. Apic. Res. Bee World. 45: 16-21. http://dx.doi.org/10.3896/ibra.1.45.2.04

Ellis, M. B. and Ellis J. P. (1997): Microfungi on land plants: an identification handbook. - Richmond Publishing, Slough, England, 860 pp.

Földházi, G. (1994): Analysis and quantitation of sugars in honey of different botanical origin using high performance liquid chromatography. - Acta Aliment. Hung. 23: 299-311.

González-Miret, M. L., Terrab, A., Hernanz, D., Fernández-Recamales, M. A. and Heredia, F. J. (2005): Multivariate correlation between color and mineral composition of honeys and by their botanical origin. - J. Agric. Food Chem. 53(7): 2574-2580. http://dx.doi. org/10.1021/jf048207p

Hughes, S. J. (1958): Deuteromycetes I: The Sporidesmium complex. - Mycologia. 50: 681692. http://dx.doi.org/10.2307/3756177

Ingold, C. T. (1971): Fungal spores. Their liberation and dispersal. - Oxford University Press, New York, 302 pp.

Kendrick, B. (1990): Fungal allergens. - In: Smith, E. G. (ed.): Sampling and identifying allergenic pollens and moulds. Blewstone Press, San Antonio, pp. 41-165.

Lacey, M. E. and West, J. S. (2006): The air spora: a manual for catching and identifying airborne biological particles. - Springer, Dordrecht, $156 \mathrm{pp}$.

Louveaux, J., Maurizio, A. and Vorwohl, G. (1978): Methods of melissopalynology. - Bee World. 51: 139-157. http://dx.doi.org/10.1080/0005772x.1978.11097714

Magyar, D. (2008): The tree bark: a natural spore trap. - Asp. Appl. Biol. 89: 7-16.

Magyar, D. and Révay, Á. (2009): New species of Oncopodiella (Hyphomycetes) from living trees. - Nova Hedwigia 88(1-2): 169-182. http://dx.doi.org/10.1127/00295035/2009/0088-0169

Magyar, D., Gönczöl, J., Révay Á., Grillenzoni, F. and Del Carmen Seijo-Coello, M. (2005): Stauro- and scolecoconidia in floral and honeydew honeys. - Fungal Divers. 20: 103-120.

Magyar, D., Shoemaker, R. A., Bobvos, J., Crous, P. W. and Groenewald, J. Z. (2011): Pyrigemmula, a novel hyphomycete genus on grapevine and tree bark. - Mycol. Prog. 10(3): 307-314. http://dx.doi.org/10.1007/s11557-010-0703-4

Peel, M. C., Finlayson, B. L. and McMahon, T. A. (2007): Updated world map of the Köppen-Geiger climate classification. - Hydrol. Earth Syst. Sci. 4(2): 439-473. http://dx.doi. org/10.5194/hessd-4-439-2007

Pérez-Atanes, S., Del Carmen Seijo-Coello, M. and Méndez-Álvarez, J. (2001): Contribution to the study of fungal spores in honeys of Galicia (NW Spain). - Grana 40: 217-222. http://dx.doi.org/10.1080/001731301317223240

Persano-Oddo, L. and d'Albore, G. R. (1989): Nomenclatura melissopalinológica. - Apicoltura 5: 63-72.

Persano-Oddo, L., Piana, M. L. and d'Albore, G. R. (2007): I mieli regionali italiani. - Ministero delle Politiche Agricole e Forestali, Roma. 
Persano-Oddo, L., Sabatini, A. G., Accorti, M., Colombo, R., Marcazzan, G. L., Piana, M. L., Piazza, M. G. and Pulcini, P. (2000): I mieli uniflorali italiani, nuove shede di caratterizzazione. - Ministero delle Politiche Agricole e Forestali, Roma.

Sabatini, A. G., Bortolotti, L. and Marcazzan, G. L. (2007): Conoscere il miele. - Ed. Avenue Media, CRA-INA di Bologna, 371 pp.

Seijo, M. C., Escuredo, O. and Fernández-González, M. (2011): Fungal diversity in honeys from northwest Spain and their relationship to the ecological origin of the product. Grana 50: 55-62. http://dx.doi.org/10.1080/00173134.2011.559555

Thrasyvoulou, A. and Manikis, J. (1995): Some physicochemical and microscopic characteristics of Greek unifloral honeys. - Apidologie 26(6): 441-452. http://dx.doi.org/10.1051/ apido:19950601

Zander, E. (1935): Herkunftsbestimmung bei Honig. - Angew. Chem. 48(9): 147-149. http:// dx.doi.org/10.1002/ange.19350480903 